\title{
Susceptibility-weighted cardiovascular magnetic resonance in comparison to T2 and T2 star imaging for detection of intramyocardial hemorrhage following acute myocardial infarction at 3 Tesla
}

Ananth Kidambi ${ }^{1}$, John D Biglands², David M Higgins ${ }^{3}$, David P Ripley ${ }^{1}$, Arshad Zaman², David A Broadbent ${ }^{2}$, Adam K McDiarmid ${ }^{1}$, Peter P Swoboda', Tarique Al Musa ${ }^{1}$, Bara Erhayiem ${ }^{1}$, John P Greenwood ${ }^{1}$ and Sven Plein ${ }^{1 *}$

\begin{abstract}
Background: Intramyocardial hemorrhage $(\mathrm{IMH})$ identified by cardiovascular magnetic resonance (CMR) is an established prognostic marker following acute myocardial infarction (AMI). Detection of IMH by T2-weighted or T2 star CMR can be limited by long breath hold times and sensitivity to artefacts, especially at 3T. We compared the image quality and diagnostic ability of susceptibility-weighted magnetic resonance imaging (SW MRI) with T2-weighted and T2 star CMR to detect IMH at 3T.

Methods: Forty-nine patients (42 males; mean age 58 years, range 35-76) underwent 3T cardiovascular magnetic resonance (CMR) 2 days following re-perfused AMI. T2-weighted, T2 star and SW MRI images were obtained. Signal and contrast measurements were compared between the three methods and diagnostic accuracy of SW MRI was assessed against T2w images by 2 independent, blinded observers. Image quality was rated on a 4-point scale from 1 (unusable) to 4 (excellent).

Results: Of 49 patients, IMH was detected in 20 (41\%) by SW MRI, 21 (43\%) by T2-weighted and 17 (34\%) by T2 star imaging ( $p=n s)$. Compared to T2-weighted imaging, SW MRI had sensitivity of $93 \%$ and specificity of $86 \%$. SW MRI had similar inter-observer reliability to T2-weighted imaging ( $k=0.90$ and $k=0.88$ respectively); both had higher reliability than T2 star $(k=0.53)$. Breath hold times were shorter for SW MRI ( 4 seconds vs. 16 seconds) with improved image quality rating $(3.8 \pm 0.4,3.3 \pm 1.0,2.8 \pm 1.1$ respectively; $p<0.01)$.
\end{abstract}

Conclusions: SW MRI is an accurate and reproducible way to detect IMH at 3T. The technique offers considerably shorter breath hold times than T2-weighted and T2 star imaging, and higher image quality scores.

Keywords: Hemorrhage, Magnetic resonance imaging, Cardiovascular magnetic resonance, Myocardial infarction, Susceptibility

\footnotetext{
* Correspondence: S.Plein@leeds.ac.uk

${ }^{1}$ Multidisciplinary Cardiovascular Research Centre \& Leeds Institute of

Cardiovascular and Metabolic Medicine, University of Leeds, LS2 9JT Leeds,

UK

Full list of author information is available at the end of the article
} 


\section{Background}

The aim of emergency treatment for ST-elevation acute myocardial infarction (AMI) is coronary reperfusion, optimally via primary percutaneous coronary intervention (PCI). In approximately $40 \%$ of patients, reperfusion by primary PCI is associated with detectable reperfusion injury [1]. Reperfusion injury may manifest as microvascular obstruction (MO), which is associated with adverse functional outcome [2] and worse prognosis [3,4]. A subset of patients with MO may also have hemorrhage within the infarcted myocardium. Intramyocardial hemorrhage (IMH) is independently associated with adverse prognosis over and above MO alone [5]. The most sensitive clinical way of detecting IMH is by cardiovascular magnetic resonance (CMR) imaging [6]. The breakdown products of hemoglobin within IMH exert a paramagnetic effect which shortens $\mathrm{T} 2$ relaxation times, resulting in the presence of a characteristic hypointense infarct core on T2-weighted (T2w) or T2* imaging [7]. However, this important prognostic marker has been relatively underused in the clinical setting. One reason for this underuse is the difficulty in obtaining reliable diagnostic quality images, which for dark-blood T2w or T2* commonly require long breath hold times ( 16 s) with minimal respiratory movement. This may be especially difficult in the context of recent acute myocardial infarction, which commonly causes breathlessness and orthopnea.

Alternative methods that can detect IMH with shorter breath hold times are therefore desirable. MR is capable of detecting differences in the magnetic susceptibility of tissues. The paramagnetic properties of hemoglobin products within IMH cause local phase shifts relative to surrounding tissue [8]. The k-space data acquired for each magnitude image can be also used to derive phase data. With the exception of large vessel flow quantification, clinical CMR largely relies on magnitude data, and phase data are mostly discarded. Phase data can be filtered and combined with magnitude data to generate susceptibility weighted MR images (SW MRI) [9]. SW MRI has the inherent potential advantage of short acquisition times, without the need for spin refocusing or multiple images. SW MRI effects may be more pronounced at 3T due to increased phase differences between tissues, whereas myocardial T2 and T2* images may be degraded at higher field strengths, in part due to these susceptibility effects [10].

SW MRI has been used clinically in neuroimaging, to visualize venous structures in the brain [11], and has been shown to be highly sensitive for the detection of cerebral hemorrhage in stroke [12]. We hypothesized that SW MRI could be used to detect hemorrhage following AMI, especially at 3T. We compared the image quality and diagnostic accuracy of SW MRI with T2w and T2* CMR at 3T for the detection of IMH following reperfused AMI.

\section{Methods}

\section{Patient selection}

Patients with first ST-segment elevation AMI, revascularized by primary PCI within 12 hours of onset of pain were prospectively recruited from a single tertiary center from February 2012 to August 2013. AMI was defined as per current guidelines [13]. Exclusion criteria were previous AMI or coronary artery bypass grafting, cardiomyopathy, estimated glomerular filtration rate $<30 \mathrm{ml} / \mathrm{min} / 1.73 \mathrm{~m}^{2}$, or contraindications to CMR. The study protocol was approved by the institutional research ethics committee (NHS Health Research Authority, NRES Leeds West) and complied with the Declaration of Helsinki; all patients gave written informed consent. Patients with maximal total scar extent (including MO or IMH) less than 2 voxels of the in-plane resolution of LGE (approximately 3$4 \mathrm{~mm}$ ) were deemed too small for accurate evaluation of the infarct zone and not included in the analysis. Clinical management (including anticoagulation and use of aspiration catheters) was performed blind to the CMR results and at the discretion of the responsible clinician, reflecting contemporary practice and guidelines. All patients were considered for angiotensin converting enzyme inhibitors, beta-blockade, statins, dual antiplatelet therapy and cardiac rehabilitation.

\section{Image acquisition}

All patients had CMR imaging at $3.0 \mathrm{~T}$ within 3 days (median 2 days) of their index presentation (Achieva TX, Philips Healthcare, Best, The Netherlands equipped with a Quasar Dual gradient system $(40 \mathrm{mT} / \mathrm{m} ; 200 \mathrm{~T} / \mathrm{m} / \mathrm{s}$ ) and radiofrequency $(\mathrm{RF})$ shimming with dual-source $\mathrm{RF}$ transmission). A dedicated 32-channel cardiac phased array receiver coil was used. Cine imaging was performed using a contiguous stack of parallel short-axis slices covering the whole left ventricle (LV), with a balanced steady-state free precession pulse sequence (echo time (TE) $1.3 \mathrm{~ms}$; repetition time (TR) $2.6 \mathrm{~ms}$; flip angle $40^{\circ}$, spatial resolution $1.6 \times 2.0 \times 10 \mathrm{~mm}, 40$ phases per cardiac cycle). SW, T2w, T2* and late gadolinium enhancement (LGE) imaging were performed using the '3-of-5' approach by acquiring the central 3 slices of 5 parallel short-axis slices spaced equally from mitral valve annulus to LV apical cap [14]. The same slice geometry, position and a $10 \mathrm{~mm}$ slice thickness were used for all pulse sequences. The SW sequence used a black-blood inversion recovery turbo gradient echo sequence (sensitivity encoding (SENSE) parallel acceleration factor 2.3, TR/TE/flip angle $4.1 \mathrm{~ms} / 3.0 \mathrm{~ms} / 20$ degrees, spatial resolution $1.8 \times 2.5 \times 10 \mathrm{~mm}$, typical matrix $212 \times 146$, pre-pulse black blood delay $775 \mathrm{~ms}$ ). Magnitude and phase images were generated online at the time of scanning. T2w imaging used a dark-blood T2w fast spin echo short tau inversion-recovery (STIR) 
sequence (TE $90 \mathrm{~ms}$, TR two R-R intervals, flip angle 90 degrees, spatial resolution $1.7 \times 1.7 \times 10 \mathrm{~mm}$, typical matrix $208 \times 200$ ) and constant level appearance (CLEAR) homogeneity correction. For $\mathrm{T}_{2}^{*}$ imaging, 32 gradient echoes were subdivided into six groups, with a linear $\mathrm{k}$-space order within each group contributing to a separate $\mathrm{k}$-space. The echoes used for the center of k-space for each image/group had consistent parity. T2* imaging parameters were as follows: SENSE $=2$, TFE factor 8 , TR/ TE1/echo spacing (ms) 15/2.3/2.2, spatial resolution $1.8 \times$ $2.5 \times 10 \mathrm{~mm}$, typical matrix $176 \times 128$, pre-pulse black blood delay $420 \mathrm{~ms}$, trigger delay set to image in late diastole. To minimize regional myocardial variation for $\mathrm{T} 2$ * imaging, image-based shimming was employed [10]. $0.1 \mathrm{mmol} / \mathrm{kg}$ gadolinium-DTPA (gadopentetate dimeglumine; Magnevist, Bayer, Berlin, Germany) was then administered using a power injector (Spectris, Solaris, PA). LGE imaging was performed at 16-20 minutes following contrast (inversion recovery-prepared T1 weighted gradient echo, inversion time according to Look-Locker scout, TR/TE/flip angle $3.7 \mathrm{~ms} / 2.0 \mathrm{~ms} / 25$ degrees, spatial resolution $1.54 \times 1.75 \times 10 \mathrm{~mm}$, typical matrix $232 \times 182$ ). Breath hold times per slice at a typical heart rate of 60 / min were: 16 seconds for $\mathrm{T} 2 \mathrm{w}, 17$ seconds for $\mathrm{T} 2$ * and 4 seconds for SW data acquisition. For each pulse sequence, images with motion or parallel imaging artefact were repeated until any artefact was removed or minimized. The highest quality images were used for analysis.

\section{Image analysis}

Phase and magnitude data were combined into a SW MRI image using the SWIp algorithm (Susceptibility Weighted Imaging with Phase enhancement; previously referred to as "PADRE") [15,16]. To enable testing of different SWIp parameters, images were processed offline, taking $<10$ seconds per image (SWIp tool v1.7, Philips Healthcare, The Netherlands). Automated inline processing on the scanner console with fixed parameters is possible with processing time $<5$ seconds. The SWIp tool calculates a contrast-enhancing mask from the phase images. The phase information can be contaminated by background field effects and so it is initially corrected by applying a homodyne high pass filter to the complex-valued image data [17]. The SWIp phase $(\phi)$ mask is defined by a function, the shape of which is controlled by three adjustable parameters, $\alpha, \beta$ and $\sigma$ :

$$
\operatorname{Mask}(x)=\left\{\begin{array}{cc}
e^{-\alpha\left(|\phi(x)|-\frac{\sigma * \pi}{100}\right)^{\beta}} & \text { if }|\phi(x)| \geq \frac{\sigma * \pi}{100} \\
1 & \text { if }|\phi(x)|<\frac{\sigma * \pi}{100}
\end{array}\right.
$$

Figure 1 shows the influence of the parameters on the phase mask. The resulting SWIp image is given by the product of the magnitude image and the SWIp mask. As there are no agreed values for $\alpha, \beta$ or $\sigma$ for IMH, the effects of varying these parameters were tested on the first 10 patients with visible IMH on T2w imaging. All possible permutations of the following were tested: filter size: $64 \times 64,128 \times 128 ; \alpha$ : $0,0.2,0.4,0.6,0.8,0.95 ; \beta: 0.1$, $0.2,0.3,0.4,0.55,0.6,0.8,1.0$; $\sigma: 0,0.5,1,2,3,5,7.5,15$, $25,50,75$. These parameter values were chosen to sample the spread of potential values, and also to focus on specific values previously reported [16]. Filter sizes were chosen to account for the larger comparative size of IMH and higher field strength than previous validation work in neuroimaging [18]. Regions of interest (ROI) were drawn using in-house software written in Matlab

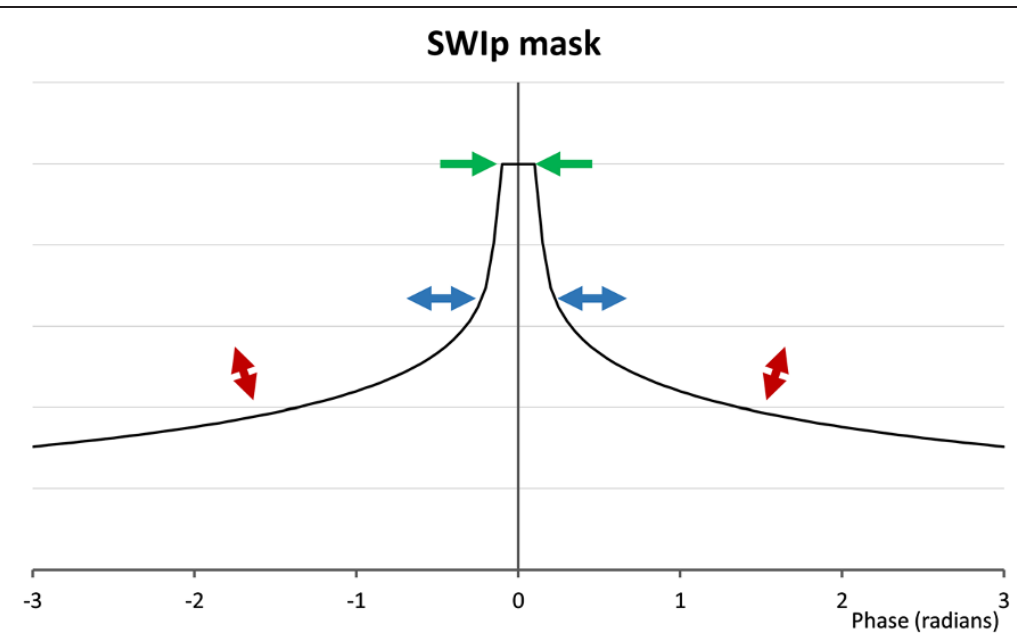

Figure 1 Effect of parameters on SWIp phase mask. A plot of mask value (y-axis) vs. phase (x-axis) is shown. a predominantly controls the gradient and height of the mask at higher absolute phase values (red arrows), $\beta$ predominantly affects the slope of the masking curve at intermediate phase values (blue arrows), and changes in $\sigma$ predominantly control masking at phase values close to zero (green arrows). 
(Mathworks, Natick, MA, version R2011b) within areas of IMH as defined on T2w imaging and remote myocardium (in myocardium opposite to the infarct zone as defined on LGE imaging and away from the infarct and peri-infarct zone). Areas of hypointensity were visualized and contoured manually. In order to compare different SWIp parameter values, mean signal intensity (SI) and standard deviation (SD) were measured for each myocardial region. The relative signal to noise (rSNR) for IMH regions was evaluated; tissue contrast was evaluated by calculating rSNR difference ( $\triangle \mathrm{rSNR})$, using the following methods [19]:

$$
\begin{aligned}
& r S N R=0.655 \times \frac{S I}{S D} \\
& \Delta r S N R=r S N R_{\text {remote }}-r S N R_{\mathrm{IMH}}
\end{aligned}
$$

The SNR calculation is subject to a number of errors including residual B1 inhomogeneity and non-uniform image noise due to parallel image reconstruction [20]. However, measurement of the absolute SNR was less critical than an accurate estimate of the difference between rSNR measurements. $\triangle \mathrm{rSNR}$ allows for a quantitative comparison of contrast generation between tissue types in SWIp images using different phase masks, assuming similar coil gain and geometry factor between infarct and IMH ROIs for the acceleration factor used.

The values of filter size, $\alpha, \beta$ and $\sigma$ that generated the highest $\triangle \mathrm{rSNR}$ were derived from the first 10 patients with visible IMH on T2w imaging, and these values then applied to all patients for the main analysis. For analysis, the phase mask was applied once, except where indicated for testing of rSNR and $\triangle \mathrm{rSNR}$ with multiple applications of the phase mask.

SWIp, T2w and T2* images were independently evaluated for the presence of IMH by two blinded reviewers (AK and DPR, three years of CMR experience). Disagreement between reviewers was resolved by a consensus read. The presented accuracy statistics are based on consensus analysis. A further blinded read was taken (by AK) more than one month after the initial read for intraobserver analysis. The presence of IMH was assessed in conjunction with LGE images, to reflect realworld practice; reviewers were blinded to other imaging sequences, patient and clinical details. IMH was considered to be present when an area of hypointensity was visible within an area corresponding to the infarct zone on LGE imaging. For rSNR analysis, signal intensity and SD were evaluated in IMH and remote myocardial regions based on the consensus read. For T2* images, all echoes were analyzed, the single image per slice with the highest $\triangle \mathrm{rSNR}$ was chosen for statistical analysis. T2w, $\mathrm{T} 2 \%$, cine and LGE images were evaluated offline using commercial software (cvi42 v4.1.5, Circle Cardiovascular Imaging Inc., Calgary, Canada). Image quality was assessed by consensus of 2 observers, and on a slice-by-slice basis according to a 4 point scale: $4=$ excellent, $3=$ minor artefact compromising diagnostic accuracy in myocardium outside of the infarct territory, 2 = artefact compromising infarct zone but analysis possible, $1=$ unusable. Left ventricular volumes and ejection fraction (EF) were analyzed from cine images using standard methods [21]. Infarct location was determined by LGE imaging, according to standard guidelines [22].

\section{Statistical analysis}

Statistical analysis was performed using IBM SPSS ${ }^{\circ}$ Statistics 21.0. Continuous variables are expressed as mean \pm SD. Normality for quantitative data was established using the Kolmogorov-Smirnov test. Demographic comparisons were performed with an independent samples $t$-test with Bonferroni correction to account for multiple comparisons. Differences in measurements per-slice were evaluated using a multilevel linear mixed-effects model to account for non-independence of slice data. Post-hoc comparisons were made using Tukey's test. Inter and intra-rater reliability were performed using Cohen's Kappa statistic. All statistical tests were 2 -tailed; $\mathrm{p}$ values $<0.05$ were considered significant.

\section{Results}

54 patients met the inclusion criteria. In 5 patients the infarct size was too small for accurate analysis as per the criteria above; therefore 49 patients were included in the statistical analysis. Patient characteristics are shown in Table 1. Myocardial characteristics are shown in Table 2. No gender-based differences in characteristics were present ( $p>0.1$ for all).

\section{Choice of image weighting parameters}

The first 10 sequential patients with IMH visible on $\mathrm{T} 2 \mathrm{w}$ imaging were selected to evaluate the optimal image weighting parameters for the SWIp sequence to detect IMH. rSNR ${ }_{I M H}$ and $\triangle \mathrm{rSNR}$ (between IMH and remote myocardium) for each combination of parameters were averaged over the 10 patients. These varied substantially depending on parameter values (rSNR: mean $3.55 \pm 0.65$, range 1.13-4.28; $\triangle \mathrm{rSNR}$ mean $0.60 \pm$ 0.25 , range $0.00-1.33$ ). The highest and lowest $\Delta \mathrm{rSNR}$ results with corresponding parameter values are shown in Table 3. The following parameters produced the highest $\triangle \mathrm{rSNR}$ (i.e. the greatest relative SNR difference between IMH and remote myocardium) and were hence applied to the SW MRI images used for analysis in the whole population: filter size $128 \times 128, \alpha=0.95$, $\beta=0.2, \sigma=3$. 
Table 1 Patient characteristics

\begin{tabular}{ll}
\hline Patient characteristic & \\
\hline $\mathrm{n}$ & 49 \\
Age, years & $58.0 \pm 11.3$ \\
Male & $42(86 \%)$ \\
Body mass index, kg/m² & $28.2 \pm 3.3$ \\
Current smoker & $27(55 \%)$ \\
Hypertension & $12(25 \%)$ \\
Hypercholesterolemia & $13(27 \%)$ \\
Diabetes mellitus & $6(12 \%)$ \\
Pain to balloon time, min (median (IQR $\left.\left.{ }^{*}\right)\right)$ & $219(275)$ \\
MO present & $25(51 \%)$ \\
TIMI flow grade $\geq 2$ pre-PCl & $4(8 \%)$ \\
TIMI flow grade 3 post PCl & $47(96 \%)$ \\
Peak troponin I, ng/L (median) & $>50000$ \\
Peak CK', iu/L (median (IQR)) & $601(1297)$ \\
Infarct territory & \\
Anterior & $22(45 \%)$ \\
Inferior & $21(43 \%)$ \\
Lateral & $6(12 \%)$ \\
\hline
\end{tabular}

Data as mean $\pm \mathrm{SD}$ or $\mathrm{n}(\%)$ unless indicated. ${ }^{*} I Q R$ interquartile range,

${ }^{\dagger} \mathrm{CK}$ creatine kinase.

\section{rSNR and $\triangle$ rSNR}

For SW MRI images, average rSNR was $3.62 \pm 2.89$ for areas of IMH and $5.61 \pm 2.63$ for remote myocardium $(\beta=$ 0.47, $\mathrm{p}<0.001) . \Delta \mathrm{rSNR}$ between remote myocardium and IMH was $1.7 \pm 2.9$. $\Delta$ rSNR between infarct and IMH was $4.71 \pm 4.48$, and between infarct and remote was $3.18 \pm$ 3.41 ( $\beta=0.87, p<0.001)$. There were no significant differences in SWIp remote rSNR dependent on ROI location (anterior 5.39 \pm 3.28 , septal $4.16 \pm 3.41$, inferior $2.46 \pm 0.68$, lateral $3.33 \pm 2.95, \beta=0.07, p=0.39$ ). Figures 2 and 3 show representative images.

For T2w images, average rSNR was $4.23 \pm 2.71$ for areas of $\mathrm{IMH}$ and $4.69 \pm 2.04$ for remote myocardium $(\beta=0.00$, $\mathrm{p}=0.99) . \triangle \mathrm{rSNR}$ between remote myocardium and IMH

Table 2 Infarct characteristics

\begin{tabular}{ll}
\hline Characteristic & Acute visit \\
\hline Ejection fraction, \% & $49 \pm 10$ \\
LV EDVi $^{*}, \mathrm{ml} / \mathrm{m}^{2}$ & $82 \pm 15$ \\
$\mathrm{LV} \mathrm{ESVi}^{\dagger}, \mathrm{ml} / \mathrm{m}^{2}$ & $42 \pm 12$ \\
LV indexed mass, $\mathrm{g} / \mathrm{m}^{2}$ & $64 \pm 14$ \\
LGE infarct volume, $\mathrm{ml}$ & $48 \pm 15$ \\
LGE MO volume, ml & $3 \pm 5$ \\
T2W IMH area (per slice, where visible), $\mathrm{mm}^{2}$ & $69 \pm 73$ \\
\hline
\end{tabular}

$\mathrm{n}=49$. Data as mean \pm SD. LV measurements are indexed to body surface area, infarct volumes are unindexed. " LV EDVi Left ventricular end diastolic volume (indexed), ${ }^{\dagger}$ LV ESVi Left ventricular end systolic volume (indexed).
Table 3 Optimal and worst susceptibility weighting parameters

\begin{tabular}{lllllll}
\hline & Filter & Alpha & Beta & Sigma & $\Delta_{\text {rSNR }}{ }^{*}$ & rSNR $^{\dagger}$ \\
\hline 1 & 128 & 0.95 & 0.20 & 3 & 1.327 & 2.115 \\
2 & 64 & 0.95 & 0.10 & 5 & 1.324 & 2.025 \\
3 & 64 & 0.95 & 0.20 & 5 & 1.321 & 2.295 \\
4 & 64 & 0.80 & 0.10 & 5 & 1.316 & 2.180 \\
5 & 128 & 0.95 & 0.10 & 5 & 1.305 & 2.702 \\
6 & 128 & 0.95 & 0.30 & 3 & 1.304 & 2.404 \\
7 & 64 & 0.95 & 0.00 & 7.5 & 1.301 & 2.605 \\
8 & 128 & 0.80 & 0.10 & 3 & 1.299 & 1.974 \\
9 & 64 & 0.80 & 0.00 & 5 & 1.299 & 1.904 \\
10 & 128 & 0.95 & 0.10 & 3 & 1.297 & 1.742 \\
1056 & 128 & 0.20 & 0.55 & 75 & 0.005 & 4.019 \\
1055 & 128 & 0.80 & 0.10 & 0.5 & 0.008 & 2.018 \\
1054 & 64 & 0.40 & 0.00 & 1 & 0.009 & 2.528 \\
1053 & 128 & 0.95 & 0.10 & 0.5 & 0.028 & 1.766 \\
1052 & 64 & 0.80 & 0.10 & 0.5 & 0.029 & 2.158 \\
1051 & 128 & 0.20 & 0.55 & 25 & 0.033 & 4.283 \\
1050 & 64 & 0.95 & 0.00 & 1 & 0.039 & 1.270 \\
1049 & 128 & 0.20 & 0.55 & 7.5 & 0.043 & 4.265 \\
1048 & 128 & 0.21 & 0.20 & 15 & 0.052 & 4.255 \\
1047 & 64 & 0.80 & 0.00 & 1 & 0.057 & 1.503 \\
\hline
\end{tabular}

$\mathrm{n}=10$. Top 10 and bottom 10 values of ${ }^{*} \Delta \mathrm{rSNR}$ difference between remote myocardium and IMH. ${ }^{\dagger} \mathrm{rSNR}$ relative signal to noise ratio of IMH. Lower rSNR values indicate more hypointense $\mathrm{IMH}$.

was $1.7 \pm 2.9$. $\triangle \mathrm{rSNR}$ between infarct and IMH was $2.20 \pm 2.27$ and between infarct and remote was $1.50 \pm$ $3.59(\beta=0.47, \mathrm{p}<0.01)$. There were no significant differences in remote rSNR dependent on ROI location (anterior $3.32 \pm 1.23$, septal $4.64 \pm 2.06$, inferior $4.60 \pm$ 2.03 , lateral $5.53 \pm 1.99, \beta=-0.06, \mathrm{p}=0.46$ ).

T2* images had average rSNR of $5.74 \pm 5.81$ for areas of IMH and $8.10 \pm 4.43$ for remote myocardium $(\beta=0.00$, $\mathrm{p}=0.99) . \Delta \mathrm{rSNR}$ between remote myocardium and IMH was $5.35 \pm 3.55$. $\triangle \mathrm{rSNR}$ between infarct and IMH was $7.29 \pm 3.34$ and between infarct and remote was $5.81 \pm$ $3.92(\beta=0.60, \mathrm{p}<0.01)$. There were no significant differences in remote rSNR dependent on ROI location (anterior $8.50 \pm 3.93$, septal $9.81 \pm 4.68$, inferior $8.90 \pm 4.45$, lateral $7.98 \pm 3.58, \beta=-0.09, \mathrm{p}=0.30)$.

There was no significant difference between SW MRI and T2w imaging for rSNR in areas of IMH $(\beta=-0.27$, $\mathrm{p}=0.5), \Delta \mathrm{rSNR}$ between remote and IMH $(\beta=-0.19$, $\mathrm{p}=0.4)$ and $\triangle \mathrm{rSNR}$ between infarct and IMH $(\beta=-0.23$, $\mathrm{p}=0.6)$. rSNR in IMH was significantly higher in T2* images than SW MRI $(\beta=0.56, p<0.01)$, with no significant difference in $\triangle \mathrm{rSNR}$ between remote and IMH $(\beta=0.35$, $\mathrm{p}=0.1)$ or $\triangle \mathrm{rSNR}$ between infarct and IMH $(\beta=-0.69$, $\mathrm{p}=0.8)$. 


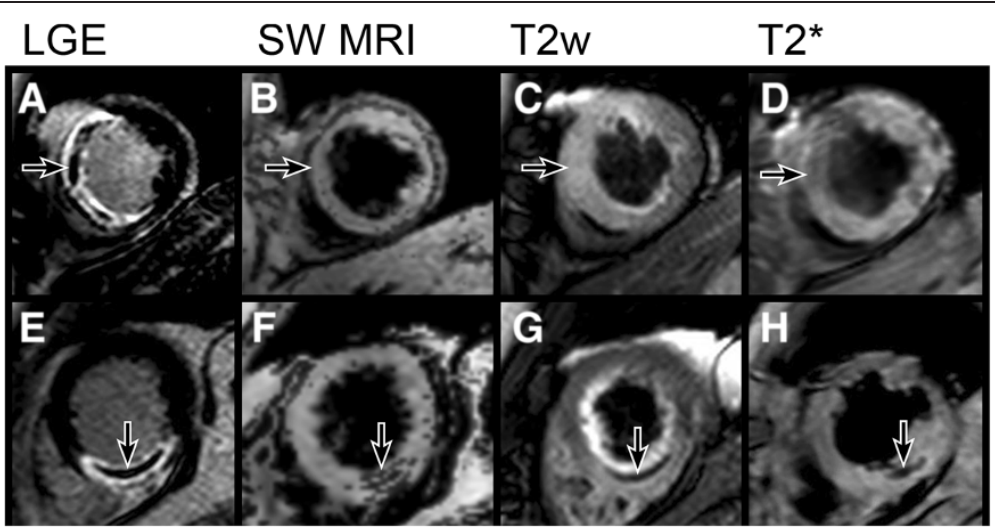

Figure 2 SW MRI comparison with T2w and T2*. Top row: MO as shown by LGE imaging ( $\mathbf{A}$, arrowed) does not correspond to hypointense myocardium indicating absence of IMH on SW MRI (B), T2W (C) and T2* (D) images. Bottom row: another patient has MO visible on LGE (E, arrowed) corresponding to hypointensity on SW MRI $(\mathbf{F}$, arrowed), T2W $(\mathbf{G}$, arrowed) and T2* $(\mathbf{H}$, arrowed) images indicating presence of IMH.

The effect of repeatedly applying the filtered phase mask to the images was evaluated. $\triangle \mathrm{rSNR}$ and rSNR measurements for each successive iteration of phase mask application are shown in Figure 4. Multiple iterations did not significantly alter $\triangle \mathrm{rSNR}$ between IMH and infarct over and above the first phase mask application $(\beta=-0.06$, $p=0.2$, Figure 4a).

\section{Image quality}

Mean image quality for SW MRI was $3.8 \pm 0.4$, for T2w was $3.3 \pm 1.0(\mathrm{p}<0.01$ compared to SW MRI) and for T2* was $2.8 \pm 1.1$ ( $\mathrm{p}<0.01$ compared to SW MRI). One (1\%) SW MRI slice, 9 (6\%) T2w slices and 30 (20\%) T2\% slices were graded as unusable (Figure 5). Of the unusable T2w slices, 6 (67\%) had clearly visible motion

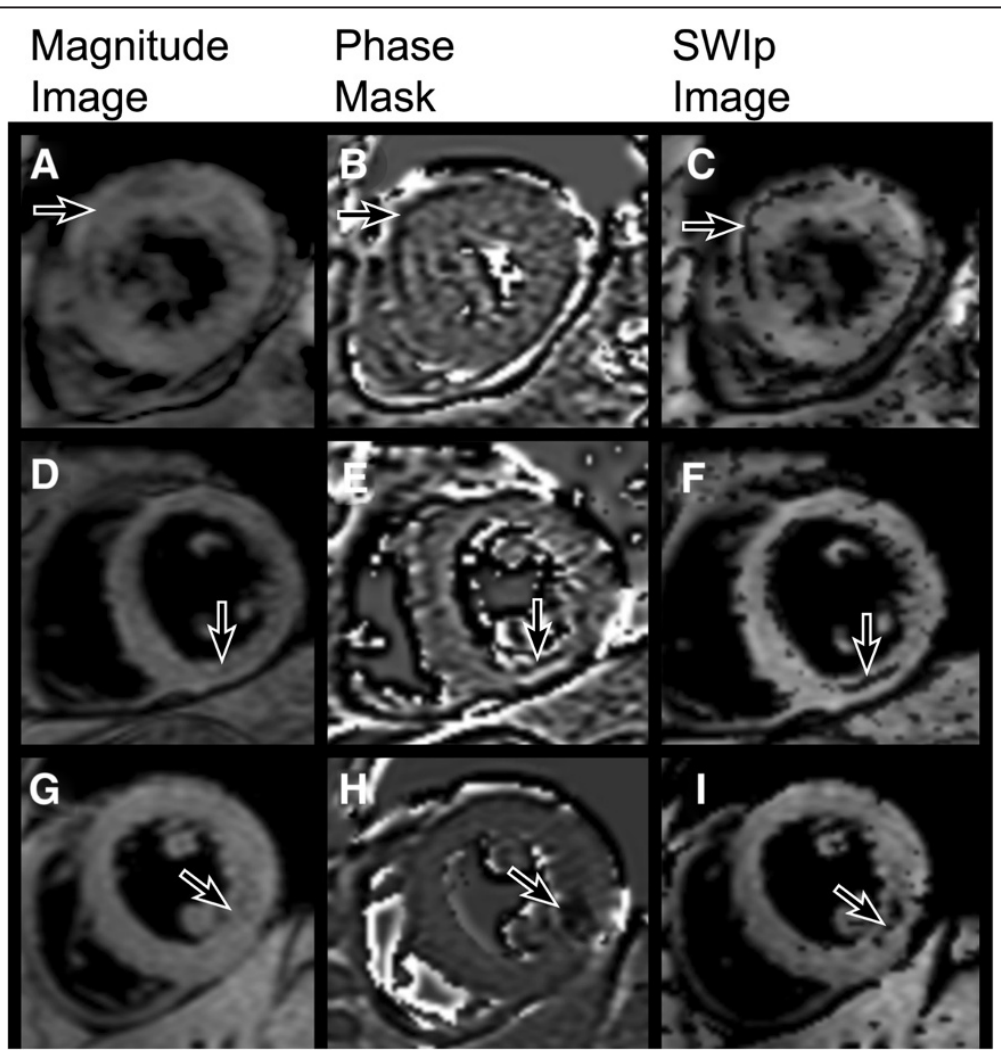

Figure 3 Contrast generation by SW MRI in three different patients. Top row: Anterior AMI with IMH (arrowed) is shown in magnitude image without susceptibility weighting (A). A phase mask is generated using the SWIp method (B) and applied to the magnitude image to generate SW images with additional contrast for IMH (C). A similar process is shown for IMH in inferior (D-F) and inferolateral (G-I) territories. 

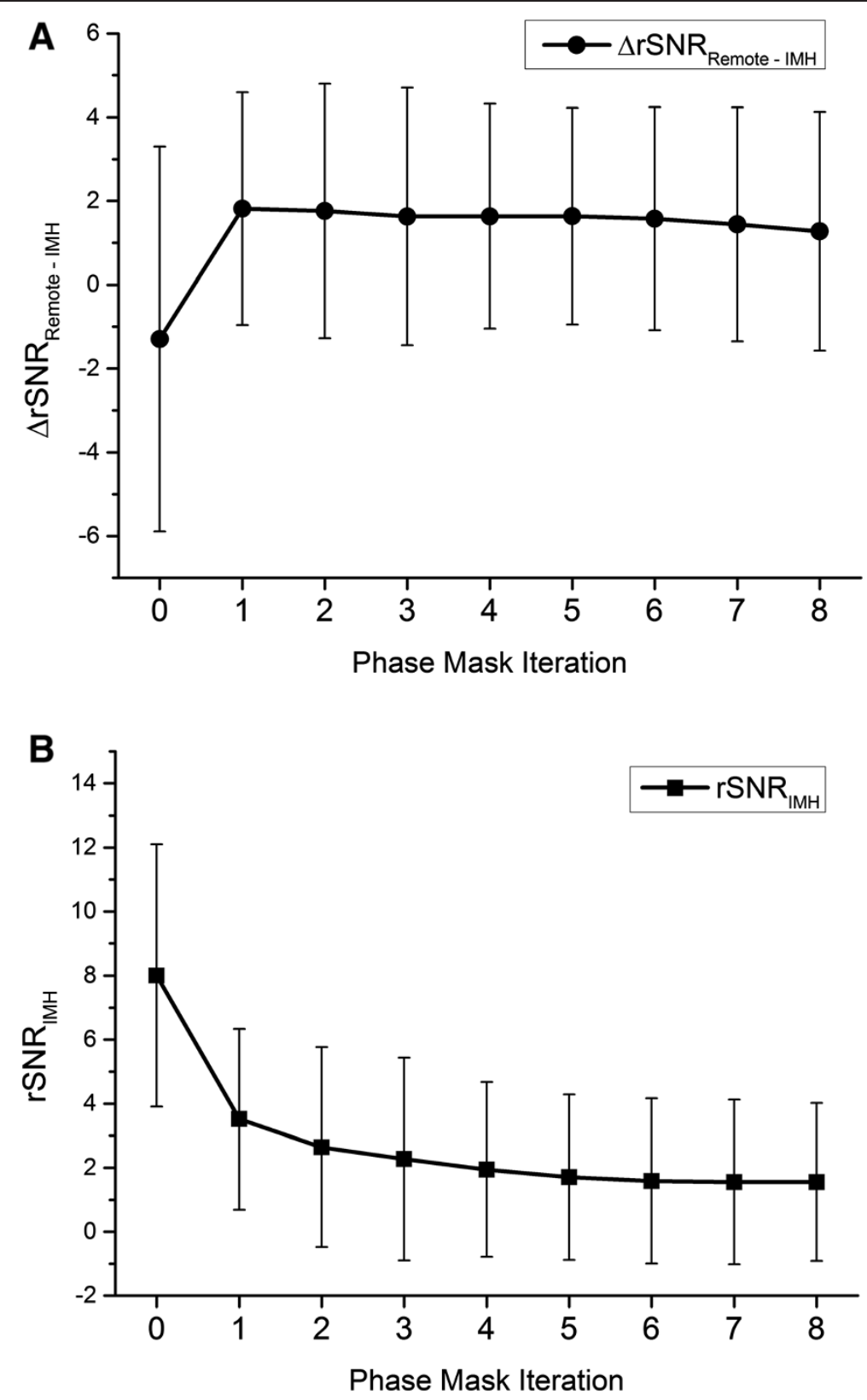

Figure 4 Effect of successive filtered phase mask applications on $\triangle$ rSNR (A) and rSNR of IMH (B). Values are averaged from all patients with IMH visible on SW MRI $(n=20)$. Iteration 0 signifies baseline images with $128 \times 128$ homodyne filter applied but no SWIp phase masking. Error bars indicate SD.

artefact. Figure 6 shows examples of optimal and suboptimal images.

\section{Image interpretation}

Per-slice, IMH within the infarct zone was detected on 34 slices $(23 \%)$ by SW MRI, 38 slices (26\%) by T2w imaging $(\mathrm{p}=0.7$ compared to SW MRI) and 31 slices (21\%) by $\mathrm{T} 2 *$ imaging ( $\mathrm{p}=0.8$ compared to SW MRI). Per-patient detection of IMH was 20 patients (41\%) by SW MRI, 21 patients (43\%) by $\mathrm{T} 2 \mathrm{w}$ ( $\mathrm{p}=1.0$ compared to SW MRI) and $17(34 \%)$ patients by $\mathrm{T} 2 * \quad(\mathrm{p}=0.68$ compared to SW MRI). Using T2w imaging as the reference standard, SW MRI had sensitivity $93 \%$ and specificity $86 \%$ on a per-patient basis, and $79 \%$ and $96 \%$ respectively on a per-slice basis. The inter-observer reliability for detection of IMH by SW MRI was $\kappa=0.82$ (95\% confidence interval $(\mathrm{CI}) 0.71-0.92)$, by $\mathrm{T} 2 \mathrm{w}$ imaging was $\kappa=0.78(0.66-0.89)$ and by $\mathrm{T} 2 \%$ imaging was $\kappa=0.53(0.36-0.69)$. Intra-observer reliability was $\kappa=0.79(0.67-0.90), \kappa=0.79(0.68-0.90)$ and $\kappa=0.74$ 


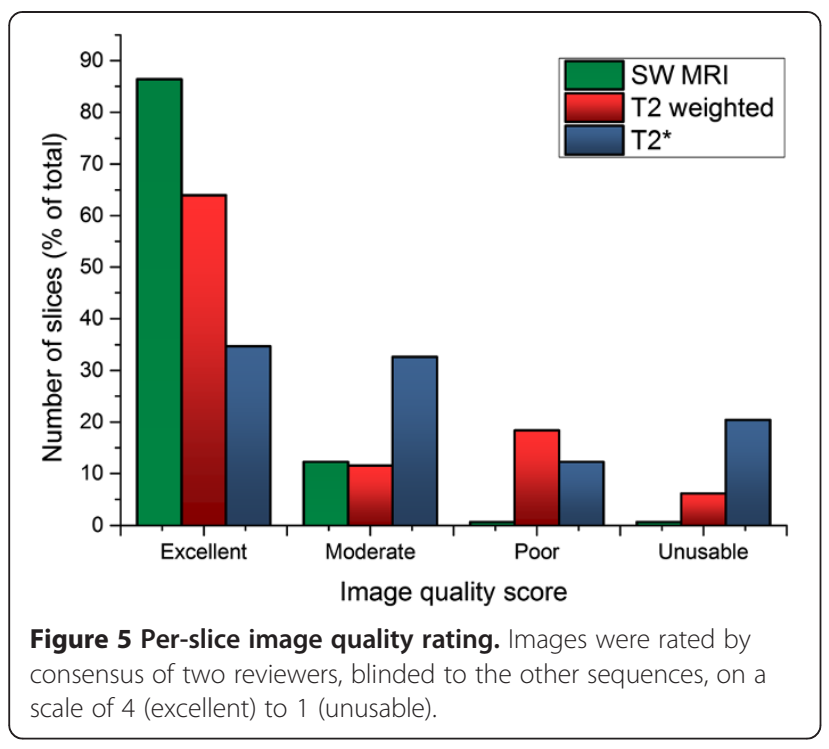

$(0.61-0.87)$ respectively for SW MRI, T2w and T2* imaging.

\section{Discussion}

This study has found that susceptibility weighted CMR at 3T, using the SWIp technique, can accurately and reproducibly identify areas of intramyocardial hemorrhage following acute myocardial infarction, with superior image quality to $\mathrm{T} 2$-weighted and $\mathrm{T}^{*}$ imaging and much shorter breath hold time.

Following reperfusion for AMI, the main clinical utility of CMR is to identify complications that affect patient prognosis. IMH is a strong marker of adverse prognosis, though the most established method of its detection,
T2w imaging, is not currently recommended as a routine part of CMR assessment in this context [23]. T2, $\mathrm{T}^{*}$ and SW MRI all rely on the paramagnetic effect of deoxygenated hemoglobin products, which in IMH will also be altered by size of hemorrhage and iron content. T2* imaging is specific for detection of IMH [7], and has been reported to be more robust than $\mathrm{T} 2$ imaging at $1.5 \mathrm{~T}$ [24]. However, at higher field strengths, increased susceptibility effects and greater $B_{1}$ magnetic field inhomogeneity substantially degrade diagnostic quality [25], whereas these effects, in part, may be utilized to enhance tissue contrast in SW MRI. T2 and T2* imaging are especially difficult post-AMI, as they are sensitive to motion and in general require long breath hold times. Free-breathing T2w and T2* techniques exist [26,27], but rely on technically complicated motion correction algorithms and, unlike SWIp, are not yet available for clinical use. Although T2* appears to have higher contrast when IMH is visible, our data show significantly lower overall image quality for $\mathrm{T} 2 \%$ imaging at $3 \mathrm{~T}$, and numerically lower detection rates for IMH as compared to SW MRI. In comparison, SW MRI magnitude data have relatively low T2-weighting, and we have shown that by integrating phase data, SW MRI provides comparable diagnostic yield to $\mathrm{T} 2 \mathrm{w}$ and $\mathrm{T} 2 *$ with much lower breath hold times (in the order of 4 seconds per slice as compared to 16-17 seconds) and superior image quality.

SW MRI offers a novel method of CMR contrast generation in addition to $\mathrm{T} 1$ or $\mathrm{T} 2$ relaxation. The technique utilizes the phase data that is acquired with each $\mathrm{k}$-space dataset, but is discarded when producing the magnitude images that are most often used clinically. By processing filtered phase data and combining it with the

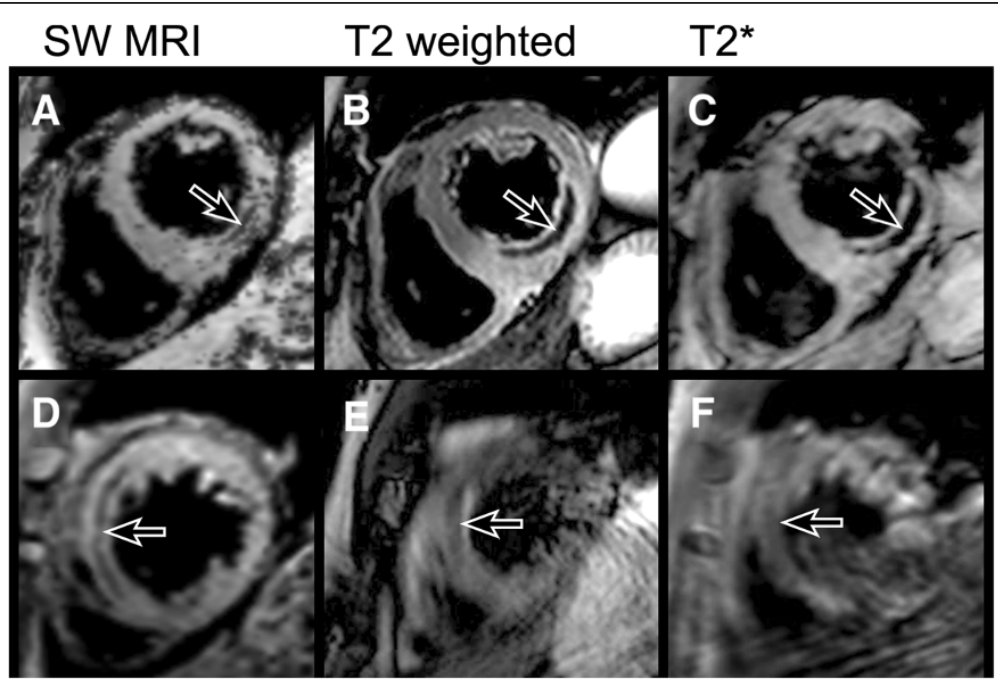

Figure 6 Optimal and suboptimal breath holding in SW MRI, T2W and T2* images. Top row: a patient with good breath holding reveals clear inferior IMH on SW MRI (A), T2W (B) and T2* (C) images. Bottom row: a different patient with anteroseptal AMI and reduced ability to breath hold clearly demonstrates IMH on SW MRI (D), but T2W (E) and T2* (F) images are suboptimal to detect IMH. 
magnitude image, an anatomical image can be created in which contrast is enhanced by the phase differences produced by deoxygenated blood products (Figure 3) [28]. Phase information can be processed in a number of different ways prior to combining with the magnitude data, and the SWIp technique offers considerable flexibility through the use of multiple parameters, whilst maintaining rapid imaging processing time $(<5$ seconds for automated inline processing). Neurological applications of SW imaging commonly use TE $>10 \mathrm{~ms}$ to generate strong T2weighting, but this would result in unacceptably long shot duration and acquisition time (e.g. $200 \mathrm{~ms}$ and $16 \mathrm{~s}$ respectively). We have shown that phase data, with appropriate filtering, can be used to help detect hemorrhage in images with relatively short TE and lower T2 weighting, with the benefit of shortened acquisition times and lower image artefacts. In this study we have defined the optimal parameters in this implementation of the SWIp phase mask to enhance IMH.

Susceptibility weighting has been most commonly used in brain imaging, with sparse literature relating to cardiac applications. It is sensitive to early detection of acute hemorrhagic stroke and microbleeds [29], and can also detect intraventricular cerebral hemorrhage in traumatic brain injury and hemorrhagic cerebral tumors [11]. Goldfarb et al. performed an analysis of 11 patients post-AMI, imaged with T2w and SW MRI, and found that phase differences in areas with IMH were significantly different to normal variations in phase difference [30]. The Goldfarb study established the feasibility of the technique, and used a pulse sequence with longer TR and TE, resulting in stronger $\mathrm{T}^{*}$ weighting and relatively long breath hold times. The sequence in the current study deliberately uses shorter echo and repetition times to ensure a shorter breath hold time but with comparable clinical utility to $\mathrm{T} 2 \mathrm{w}$ and $\mathrm{T}^{*}$ imaging. Image quality was higher for SW MRI, with an increased proportion of studies without artefact (Figures 5 and 6).

With SWIp, areas of IMH generally often appear as layers rather than a continuous region of reduced signal (Figures 2F and 6A). It is not clear whether this reflects higher spatial discrimination of SW MRI, or whether it is due to the differences between SW MRI and T2w or T2* imaging. Animal studies (such as [31]) typically describe IMH as confluent, macroscopic areas. However, IMH could potentially occur in small volumes of myocardium, below the detection level of $\mathrm{T} 2 \mathrm{w}$ or $\mathrm{T} 2 * \mathrm{im}-$ aging, or tissue may contain heterogeneous areas of IMH. SW MRI may be sensitive to phase changes between small-volume structures and could thus potentially detect smaller areas of IMH, but this clinical study could not test this assumption. Clinically, only the presence and not the size of IMH has been associated with adverse outcome. In the present study, we also did not attempt to quantify the size or severity of IMH, because the amount of signal hypointensity within IMH is related to both iron concentration and oxygenation status of hemoglobin.

Artefact at the 'heart-lung-liver' interface at the inferolateral wall was typically much less with SW MRI than with T2* imaging (Figure 6, top row). The optimal parameters derived in this study did result in some aliased pixels in the phase images being carried into the SWIp images. However, these hypointense pixels are clearly distinguishable from true IMH in their small size and position away from the infarct zone (Figure 3).

It has been suggested that multiple phase mask multiplications help to increase the visibility of small areas of hemorrhage in SW brain imaging [9]. We evaluated the impact of multiple SWIp phase mask multiplications on $\triangle \mathrm{rSNR}$ and rSNR for areas of IMH. In contrast to a previous study [9], we found only a small numerical improvement in $\triangle \mathrm{rSNR}$ between areas of IMH and infarct between 1 and 2 phase mask applications (Figure 4); however, multiple iterative phase mask applications did not result in a significant improvement in $\triangle \mathrm{rSNR}$ over the first application.

This study has a number of potential limitations. Other pulse sequences may detect IMH, such as T1 or T2 mapping [24,32], and were not tested in this study; however $\mathrm{T} 2 \mathrm{w}$ imaging remains the reference standard with established prognostic utility following AMI [5]. T2w imaging may provide other insights post-AMI, which SW MRI does not, such as estimation of the area at risk and myocardial salvage [33,34]. The SNR of SW MRI, with the mask parameters provided, is likely to change at different field strengths, with the potential to alter diagnostic accuracy.

\section{Conclusions}

SW MRI, using the SWIp technique, is an accurate and reproducible way to detect hemorrhage following AMI at 3T. The technique offers considerably shorter breath hold times to $\mathrm{T} 2 \mathrm{w}$ and $\mathrm{T} 2 *$ imaging, and does not appear to be as prone to image artefacts.

\section{Abbreviations}

AMI: Acute myocardial infarction; EF: Ejection fraction; IMH: Intramyocardial hemorrhage; LGE: Late gadolinium enhancement; LV: Left ventricle;

MO: Microvascular obstruction; MR: Magnetic resonance; PCl: Percutaneous coronary intervention; RF: Radiofrequency; rSNR: Relative signal to noise; SD: Standard deviation; SW: Susceptibility weighted; SWIp: Susceptibility Weighted Imaging with Phase enhancement; T: Tesla; T2w: T2-weighted; T2*: T2 star.

\section{Competing interests}

The authors declare that they have no competing interests. SP is funded by a British Heart Foundation fellowship (FS/1062/28409). David M Higgins is an employee of Philips. 


\section{Authors' contributions}

AK: Conception and design, analysis and interpretation of data, drafting of manuscript. JDB: Analysis methods, interpretation of data. DMH: Interpretation of data, critical and intellectual revision of manuscript. DPR: Interpretation of data, critical and intellectual revision of manuscript. AZ Analysis methods. DAB: Critical and intellectual revision of manuscript. AKM: Critical and intellectual revision of manuscript. PPS: Critical and intellectual revision of manuscript. TAM: Critical and intellectual revision of manuscript. BE: Critical and intellectual revision of manuscript. JPG: Interpretation of data, critical and intellectual revision of manuscript. SP: Conception and design, interpretation of data, drafting of manuscript. All authors have given approval of this manuscript for publication.

\section{Acknowledgements}

We thank Gavin Bainbridge, Caroline Richmond, Margaret Saysell and Petra Bijsterveld for their invaluable assistance in recruiting and collecting data for this study.

\section{Author details}

${ }^{1}$ Multidisciplinary Cardiovascular Research Centre \& Leeds Institute of Cardiovascular and Metabolic Medicine, University of Leeds, LS2 9JT Leeds, UK. ${ }^{2}$ Division of Medical Physics \& Multidisciplinary Cardiovascular Research Centre, Leeds Institute of Cardiovascular and Metabolic Medicine, University of Leeds, Leeds, UK. ${ }^{3}$ Philips Healthcare, Philips Centre, Guildford, Surrey, UK.

Received: 25 June 2014 Accepted: 23 September 2014

Published online: 28 October 2014

\section{References}

1. Mather AN, Lockie T, Nagel E, Marber M, Perera D, Redwood S, Radjenovic A, Saha A, Greenwood JP, Plein S. Appearance of microvascular obstruction on high resolution first-pass perfusion, early and late gadolinium enhancement CMR in patients with acute myocardial infarction. J Cardiovasc Magn Reson. 2009; 11:33.

2. Kidambi A, Mather AN, Motwani M, Swoboda P, Uddin A, Greenwood JP, Plein $\mathrm{S}$. The effect of microvascular obstruction and intramyocardial hemorrhage on contractile recovery in reperfused myocardial infarction: insights from cardiovascular magnetic resonance. J Cardiovasc Magn Reson. 2013; 15:58.

3. de Waha S, Desch S, Eitel I, Fuernau G, Lurz P, Leuschner A, Grothoff M, Gutberlet M, Schuler G, Thiele H. Relationship and prognostic value of microvascular obstruction and infarct size in ST-elevation myocardial infarction as visualized by magnetic resonance imaging. Clin Res Cardiol. 2012; 101:487-95.

4. Wu KC, Zerhouni EA, Judd RM, Lugo-Olivieri CH, Barouch LA, Schulman SP, Blumenthal RS, Lima JA. Prognostic significance of microvascular obstruction by magnetic resonance imaging in patients with acute myocardial infarction. Circulation. 1998; 97:765-72.

5. Eitel I, Kubusch K, Strohm O, Desch S, Mikami Y, de Waha S, Gutberlet M, Schuler G, Friedrich MG, Thiele H. Prognostic value and determinants of a hypointense infarct core in T2-weighted cardiac magnetic resonance in acute reperfused ST-elevation-myocardial infarction. Circ Cardiovasc Imaging. 2011; 4:354-62.

6. Kumar A, Green JD, Sykes JM, Ephrat P, Carson JJ, Mitchell AJ, Wisenberg G, Friedrich MG. Detection and quantification of myocardial reperfusion hemorrhage using T2*-weighted CMR. JACC Cardiovasc Imaging. 2011; 4:1274-83.

7. Mather AN, Fairbairn TA, Ball SG, Greenwood JP, Plein S. Reperfusion haemorrhage as determined by cardiovascular MRI is a predictor of adverse left ventricular remodelling and markers of late arrhythmic risk. Heart. 2011; 97:453-59.

8. Weisskoff RM, Kiihne S. MRI susceptometry: image-based measurement of absolute susceptibility of MR contrast agents and human blood. Magn Reson Med. 1992; 24:375-83.

9. Haacke EM, Xu Y, Cheng YC, Reichenbach JR. Susceptibility weighted imaging (SWI). Magn Reson Med. 2004; 52:612-18.

10. Zaman A, Higgins DM, Motwani M, Kidambi A, Kouwenhoven M, Kozerke S, Greenwood JP, Plein S. Robust myocardial T2 and T2 * mapping at 3T using image-based shimming. J Magn Reson Imaging. 2014, http://dx.doi. org/10.1002/jmri.24636. [Epub ahead of print].
11. Mittal S, Wu Z, Neelavalli J, Haacke EM. Susceptibility-weighted imaging: technical aspects and clinical applications, part 2. AJNR Am J Neuroradiol. 2009; 30:232-52.

12. Akter M, Hirai T, Hiai Y, Kitajima M, Komi M, Murakami R, Fukuoka H, Sasao A, Toya R, Haacke EM, Takahashi M, Hirano T, Kai Y, Morioka M, Hamasaki K, Kuratsu J, Yamashita Y. Detection of hemorrhagic hypointense foci in the brain on susceptibility-weighted imaging clinical and phantom studies. Acad Radiol. 2007; 14:1011-19.

13. Thygesen $K$, Alpert JS, Jaffe AS, Simoons ML, Chaitman BR, White HD, Jacobs AK, Jaffe AS, Katus HA, Apple FS, Lindahl B, Morrow DA, Chaitman BR, Clemmensen PM, Johanson P, Hod H, Underwood R, Bax JJ, Bonow JJ, Pinto F, Gibbons RJ, Fox KA, Atar D, Newby LK, Galvani M, Hamm CW, Uretsky BF, Steg PG, Wijns W, Bassand JP, et al. Third universal definition of myocardial infarction. J Am Coll Cardiol. 2012; 60:1581-98.

14. Messroghli DR, Bainbridge GJ, Alfakih K, Jones TR, Plein S, Ridgway JP, Sivananthan MU. Assessment of regional left ventricular function: accuracy and reproducibility of positioning standard short-axis sections in cardiac MR imaging. Radiology. 2005; 235:229-36.

15. Ide S, Kakeda S, Korogi Y, Yoneda T, Nishimura J, Sato T, Hiai Y, Ohnari N, Takahashi M, Hachisuka K, Fujiwara H, Matsuyama A. Delineation of optic radiation and stria of Gennari on high-resolution phase difference enhanced imaging. Acad Radiol. 2012; 19:1283-89,

16. Kakeda S, Korogi Y, Yoneda T, Nishimura J, Sato T, Hiai Y, Ohnari N, Okada K, Hayashi H, Matsusue E, Uozumi T, Tsuji S. A novel tract imaging technique of the brainstem using phase difference enhanced imaging: normal anatomy and initial experience in multiple system atrophy. Eur Radiol. 2011; 21:2202-10.

17. Noll DC, Nishimura DG, Macovski A. Homodyne detection in magnetic resonance imaging. IEEE Trans Med Imaging. 1991; 10:154-63.

18. Haacke EM, Ayaz M, Khan A, Manova ES, Krishnamurthy B, Gollapalli L, Ciulla C, Kim I, Petersen F, Kirsch W. Establishing a baseline phase behavior in magnetic resonance imaging to determine normal vs. abnormal iron content in the brain. J Magn Reson Imaging. 2007; 26:256-64.

19. Kaufman $L$, Kramer DM, Crooks LE, Ortendahl DA. Measuring signal-to-noise ratios in MR imaging. Radiology. 1989; 173:265-67.

20. Pruessmann KP, Weiger M, Scheidegger MB, Boesiger P. SENSE: sensitivity encoding for fast MRI. Magn Reson Med. 1999; 42:952-62.

21. Schulz-Menger J, Bluemke DA, Bremerich J, Flamm SD, Fogel MA, Friedrich MG, Kim RJ, von Knobelsdorff-Brenkenhoff F, Kramer CM, Pennell DJ, Plein S, Nagel E. Standardized image interpretation and post processing in cardiovascular magnetic resonance: Society for Cardiovascular Magnetic Resonance (SCMR) board of trustees task force on standardized post processing. J Cardiovasc Magn Reson. 2013; 15:35.

22. Cerqueira MD, Weissman NJ, Dilsizian V, Jacobs AK, Kaul S, Laskey WK, Pennell DJ, Rumberger JA, Ryan T, Verani MS, American Heart Association Writing Group on Myocardial S, Registration for Cardiac I. Standardized myocardial segmentation and nomenclature for tomographic imaging of the heart. A statement for healthcare professionals from the Cardiac Imaging Committee of the Council on Clinical Cardiology of the American Heart Association. Circulation. 2002; 105:539-42.

23. Kramer CM, Barkhausen J, Flamm SD, Kim RJ, Nagel E, Society for Cardiovascular Magnetic Resonance Board of Trustees Task Force on Standardized Protocols. Standardized cardiovascular magnetic resonance (CMR) protocols 2013 update. I Cardiovasc Magn Reson. 2013; 15:91.

24. Kali A, Tang RL, Kumar A, Min JK, Dharmakumar R. Detection of acute reperfusion myocardial hemorrhage with cardiac MR imaging: T2 versus T2. Radiology. 2013; 269:387-95.

25. Guo H, Au WY, Cheung JS, Kim D, Jensen JH, Khong PL, Chan Q, Chan KC, Tosti C, Tang H, Brown TR, Lam WW, Ha SY, Brittenham GM, Wu EX. Myocardial T2 quantitation in patients with iron overload at 3 Tesla. J Magn Reson Imaging. 2009; 30:394-400.

26. Kellman P, Aletras AH, Mancini C, McVeigh ER, Arai AE. T2-prepared SSFP improves diagnostic confidence in edema imaging in acute myocardial infarction compared to turbo spin echo. Magn Reson Med. 2007; 57:891-97.

27. Jin N, Jolly M, Simonetti OP. Free-breathing myocardial $\mathrm{T}^{*}$ mapping using single-shot GRE-EPI and automatic non-rigid motion correction. Proc Intl Soc Mag Reson Med [Abstract]. 2014; 22.

28. Haacke EM, Mittal S, Wu Z, Neelavalli J, Cheng YC. Susceptibility-weighted imaging: technical aspects and clinical applications, part 1. AJNR Am J Neuroradiol. 2009; 30:19-30. 
29. Hermier M, Nighoghossian N. Contribution of susceptibility-weighted imaging to acute stroke assessment. Stroke. 2004; 35:1989-94.

30. Goldfarb JW, Hasan U, Zhao W, Han J. Magnetic resonance susceptibility weighted phase imaging for the assessment of reperfusion intramyocardial hemorrhage. Magn Reson Med. 2013; 71:1210-1220.

31. Fishbein MC, Y-Rit J, Lando U, Kanmatsuse K, Mercier JC, Ganz W. The relationship of vascular injury and myocardial hemorrhage to necrosis after reperfusion. Circulation. 1980; 62:1274-79.

32. Pedersen SF, Thrysoe SA, Robich MP, Paaske WP, Ringgaard S, Botker HE, Hansen ES, Kim WY. Assessment of intramyocardial hemorrhage by T1-weighted cardiovascular magnetic resonance in reperfused acute myocardial infarction. J Cardiovasc Magn Reson. 2012; 14:59.

33. Kidambi A, Mather AN, Swoboda P, Motwani M, Fairbairn TA, Greenwood $J P$, Plein $S$. Relationship between myocardial edema and regional myocardial function after reperfused acute myocardial infarction: an MR imaging study. Radiology. 2013; 267:701-8.

34. Eitel I, Desch S, Fuernau G, Hildebrand L, Gutberlet M, Schuler G, Thiele H. Prognostic significance and determinants of myocardial salvage assessed by cardiovascular magnetic resonance in acute reperfused myocardial infarction. J Am Coll Cardiol. 2010; 55:2470-79.

doi:10.1186/s12968-014-0086-9

Cite this article as: Kidambi et al:: Susceptibility-weighted cardiovascular magnetic resonance in comparison to $\mathrm{T} 2$ and $\mathrm{T} 2$ star imaging for detection of intramyocardial hemorrhage following acute myocardial infarction at 3 Tesla. Journal of Cardiovascular Magnetic Resonance 2014 16:86.

\section{Submit your next manuscript to BioMed Central and take full advantage of:}

- Convenient online submission

- Thorough peer review

- No space constraints or color figure charges

- Immediate publication on acceptance

- Inclusion in PubMed, CAS, Scopus and Google Scholar

- Research which is freely available for redistribution 